\title{
On Supporting Identification in a Hand-Based Biometric Framework
}

\author{
Pei-Fang Guo ${ }^{1}$, Prabir Bhattacharya ${ }^{2}$, and Nawwaf Kharma ${ }^{1}$ \\ ${ }^{1}$ Electrical \& Computer Engineering, Concordia University, 1455 de Maisonneuve Blvd., \\ Montreal, QC H3G 1M8, Canada \\ \{pf_guo, kharma\}ece.concordia.ca \\ ${ }^{2}$ Computer Science Department, University of Cincinnati, 814 Rhodes Hall, Cincinnati, \\ OH 45221-0030, USA \\ bhattapr@ucmail.uc.edu
}

\begin{abstract}
Research on hand features has drawn considerable attention to the biometric-based identification field in past decades. In this paper, the technique of the feature generation is carried out by integrating genetic programming and the expectation maximization algorithm with the fitness of the mean square error measure (GP-EM-MSE) in order to improve the overall performance of a hand-based biometric system. The GP program trees of the approach are utilized to find optimal generated feature representations in a nonlinear fashion; derived from EM, the learning task results in the simple kmeans problem that reveals better convergence properties. As a subsequent refinement of the identification, GP-EM-MSE exhibits an improved capability which achieves a recognition rate of $96 \%$ accuracy by using the generated features, better than the performance obtained by the selected primitive features.
\end{abstract}

Keywords: Feature generation, biometric identification, genetic programming, the expectation maximization algorithm, mean square error, classification.

\section{Introduction}

Biometrics-based identification is a verification approach using biological features in each individual. Hand features have been widely used in designing a biometric identification system and the challenge has been established [1], [2].

In pattern recognition, the analytical selection of features and the automatic generation of features provide two distinct approaches, because they rely on different sources of information [3]. It may be useful to explore new biometric identification systems that combine both methods of the feature selection and generation. In this study, building upon our previous work of the feature selection on hand images, we present an automatic method of the feature generation, using genetic programming and the expectation maximization algorithms with the mean square error measure (GP-EM-MSE) for a hand-based biometric identification system. The benefits from the combined system of the feature selection and generation are as follows: 
- to increase the probability that the errors of the individual feature selection may be compensated for by the correct results of automatic feature generation.

- to raise the contribution that the overall performance of the classification would make toward a higher recognition rate by the improvement of the quality of feature representations.

The organization of the paper is as follows: Section 2 describes briefly our previous work on the feature selection for hand images. In Section 3, GP and the $k$-means problem via EM are described as the basis of the platform, including the evaluation phase. Section 4 presents identification results, and conclusion and future work are given in Section 5.

\section{Previous Work on the Feature Selection for Hand Images}

Previous work on hand images can be categorized as the unsupervised feature selection for clustering hand images. The main tool for accomplishing this was a genetic algorithm (GA). The method, named cooperative coevolutionary clustering algorithm (CCCA), was designed to search for a proper number (without prior knowledge of it) of clusters of hand images, and simultaneously to achieve the feature selection.

CCCA was implemented using 100 hand images as the test set; see a sample of a hand image in Fig.1. The results showed that the dimensionality of the clustering space was reduced from 84 original features to 41 selected features (11 geometric and 30 statistical features), with 4 clusters produced. At the end, the output clusters were labeled with the number of input image patterns per class, assigned to each cluster. The details of the CCCA method appear in our previous work [4], [5].

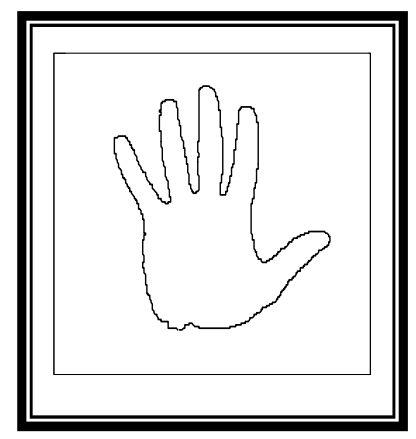

Fig. 1. A sample of a hand image

\section{The GP-EM-MSE Biometric Identification System}

The pre-requisite for the technique of the feature generation is the preparation of primitive feature sets. Using our previous method in [4] on a dataset of 200 hand images, we succeeded in clustering the 200 images into 4 categories, with a total 
number of 41 features selected as the primitive feature set for the current study of the feature generation.

Fig. 2 presents the method of the feature generation by integrating GP and EM with the MSE fitness indicator (GP-EM-MSE). In the approach, GP program trees can be viewed as sequences of applications of functions (replaced with mathematic operators) to arguments (replaced with 41 primitive features), which satisfy requirements for rational expressions of the generated features in a straightforward way; via EM, the learning task results in a simple hypothesis of the $k$-means problem. In the next subsections, we provide descriptions of each component of the biometricbased GP-EM-MSE system, including the steps of the computation.

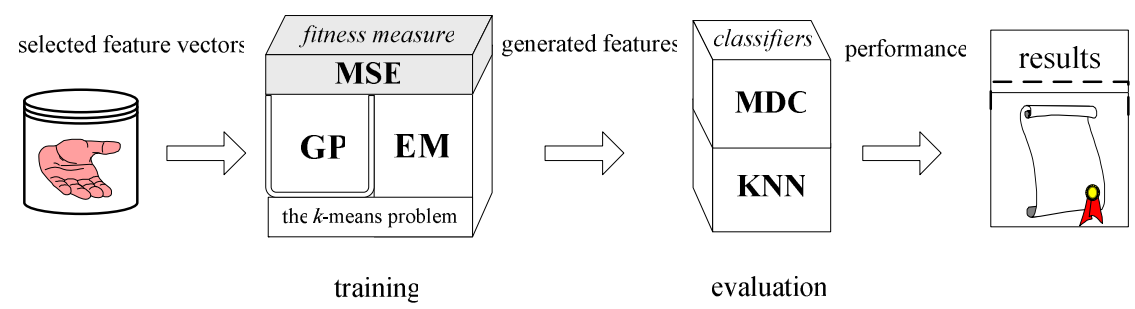

Fig. 2. The GP-EM-MSE hand-based biometric identification system

\subsection{The GP Application}

GP maintains a population of individuals by program trees. In each iteration, it produces a new generation of individuals using reproduction, crossover, and mutation. The fitness of a given individual program in the population is typically determined by executing the program on a set of the training data. The iteration of GP is illustrated in Fig. 3; the details of the GP process can be found in [6].

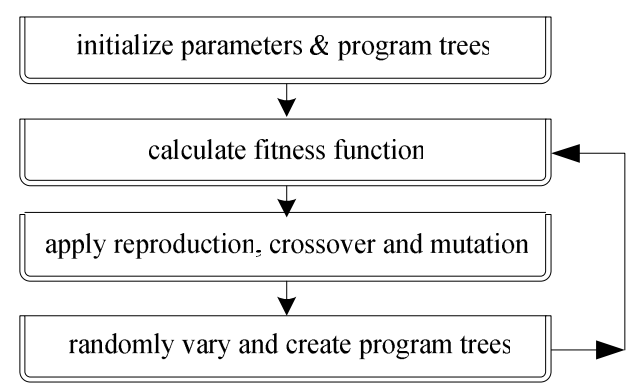

Fig. 3. The diagram of the evolutionary GP iteration

\section{The GP program trees in the approach}

The choices of terminal arguments and functions provide particular representations for describing GP program trees in the problem domain. In this study, we choose the following mathematical operators: 
$\{+,-, \times, \div$, square root, sine, cosine, tan, exponential, absolute, square, negative $\}$, which constitute the GP function set; terminal arguments receive 41 primitive features selected by the method [4].

As shown in Fig.4, an example of the rational expression, $h 2 \times h 5-\operatorname{tg}(h 5+h 8)$, can be expressed as the GP program tree, On the tree, the sign of ' $\operatorname{tg}$ ' is a mathematical operator that takes one terminal argument, and the signs of '+', '-' and ' $\mathrm{X}$ ' are operators that need two arguments. The argument, $h \mathrm{~d}, \mathrm{~d}=2,5,8$, is replaced with the $(d+1)$ th primitive feature.

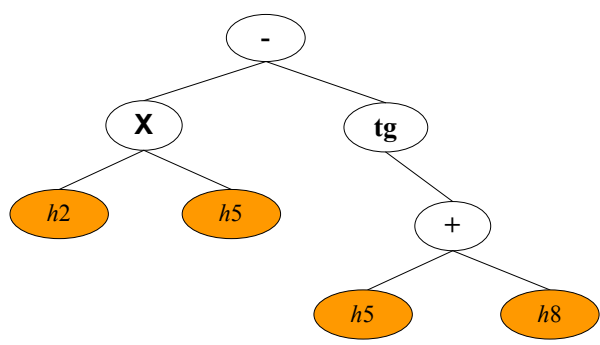

Fig. 4. A GP program tree for the rational expression of $h 2 \times h 5-\operatorname{tg}(h 5+h 8)$

\section{The fitness function, $M S E$}

The MSE measure is well known in the function approximation and learning system theories when the number of classes is assumed to be a prior known. In this supervised problem of the feature generation, we employ the MSE measure as the fitness which is given by [7]:

$$
\text { the MSE fitness }=\sum_{j=1}^{k} \sum_{i=1}^{m} D\left(c_{j}, r_{i}^{j}\right),
$$

where $D$ is the Euclidean distance between the instance $r_{i}$ and its mean center $c_{\mathrm{j}}$.

\subsection{Derivative of the $k$-Means Problem via EM}

Given the observed data $\mathbf{R}=\left\{r_{i}\right\}$, divided into the $k$ known classes; the hidden variables $\mathbf{Z}=\left\{\left\langle z_{i l}, \ldots, z_{i j}, \ldots, z_{i k}\right\rangle\right\}$ in this case indicate the index of the jth mixture component that generates $r_{i}$. With the approximation, the data $\mathbf{R}$ can be described in the mixture of the $k$ Gaussians. The purpose is to fit the Gaussian density mixture to the data $\mathbf{R}$ by optimizing the Gaussians mixture parameters, which include the mixing proportion, the mean values, and the covariance matrix. In practice, the most common use is to assume the equal mixing proportion $(1 / k)$ with a univariate case for all the $k$ categories [8], from which it simplifies the task to a hypothesis of the $k$-means problem [9]. Applying the maximum likelihood estimator, the $k$-means problem is broken down into two separate steps:

$$
\text { E-step }: E\left[z_{i j}\right]=\exp \left(-\frac{1}{2 \sigma^{2}}\left(r_{i}-\mu_{\mathrm{j}}\right)^{2}\right) / \sum_{n=1}^{k} \exp \left(-\frac{1}{2 \sigma^{2}}\left(r_{i}-\mu_{\mathrm{n}}\right)^{2}\right) \text {, }
$$




$$
M-\text { step }: \mu_{\mathrm{j}}{ }^{\prime} \leftarrow \sum_{i=1}^{m}\left[\boldsymbol{z}_{i j}\right]_{i} / \sum_{i=1}^{m} E\left[z_{i j}\right],
$$

where $\mu_{\mathrm{j}}$ is the mean value of the jth Gaussian, and $E\left[z_{i j}\right]$ is the probability that $r_{i}$ is generated by the jth Gaussian distribution. Further details can be found in [9], [10], regarding the derivative of the $k$-means problem via EM.

In the implementation, the data $\mathbf{R}$ are replaced with the generated data produced by GP-EM-MSE. The EM steps, expressed in Eqs. (2) and (3), are executed on the data $\mathbf{R}$, and constitute a joint loop with the GP iteration.

\subsection{The GP-EM-MSE Recursive Computation}

The steps of the GP-EM-MSE computation are summarized in Table 1.

Table 1. The GP-EM-MSE recursive computation

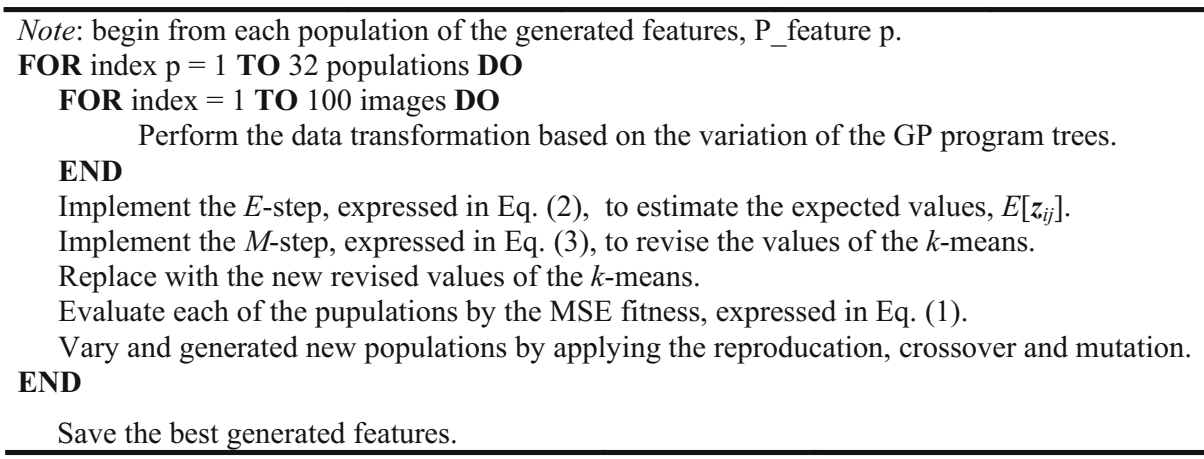

\subsection{The Evaluation Phase Using the Classifiers MDC and KNN}

The task of the identification is to classify samples into categories so that samples in the same categories are as similar as possible and samples in different categories are as dissimilar as possible. Mathematically, the problem of classification can be formulated in terms of a predetermined measure [11]. For the minimum distance classifier (MDC), the distance is defined as an index of similarity so that the minimum distance is identical to the maximum similarity; for the $\mathrm{K}$ nearest neighbors $(\mathrm{KNN})$, each image pattern is classified to the frequent class among its neighbors based on a similarity measure. In this study, the Euclidean distance measure is employed for both MDC and $\mathrm{KNN}$ in the evaluation.

\section{Identification Experiments}

In the implementation, we divided the 200 images into the training and testing sets, each with 100 images. Using the 41 primitive features as the input, the GP-EM-MSE training is evolved with the maximum tree depth of 5 and the population size of 32 . 


\subsection{Feature Generated Results}

We ended the GP-EM-MSE training after running 400 iterations. The total time for computation was about three minutes on a Pentium 4 at $1.60 \mathrm{GHz}$. The results for the two features, $P_{-}$feature 1 and $P_{-}$feature 2, produced by GP-EM-MSE are as follows:

$$
\begin{array}{cc}
P_{-} \text {feature } \quad \begin{aligned}
1= & \cos \{\sin [h 0 \times h 39 \times \operatorname{tg}(h 22 \times h 21)] \times \operatorname{tg}[|h 32 \times h 0|+h 39 \times h 27] \\
& \left.+\left|\sin \left[(h 30 / h 7)^{2}-e^{h 2 \times h 19}\right]-(h 12 \times h 31+h 14 \times h 26)^{2}\right|\right\}
\end{aligned} \\
P_{-} \text {feature } 2=\cos \left(\frac{\cos [-(h 32+h 25) \times(h 9+h 28)]}{\left|\frac{h 25}{h 23}+\cos \left(\frac{h 9}{h 8}\right)\right| \times \sqrt{\frac{h 8}{h 22 \times \sin (h 25+h 32)}+\frac{h 10}{h 8}+\cos (h 35 \times h 20)}}\right),
\end{array}
$$

where $h \mathrm{~d}$ is the $(\mathrm{d}+1)$ th feature of 41 primitive features, and the convergence results are presented in Fig. 5; in the sequel, the resulting generated features will be employed to identify hand images.

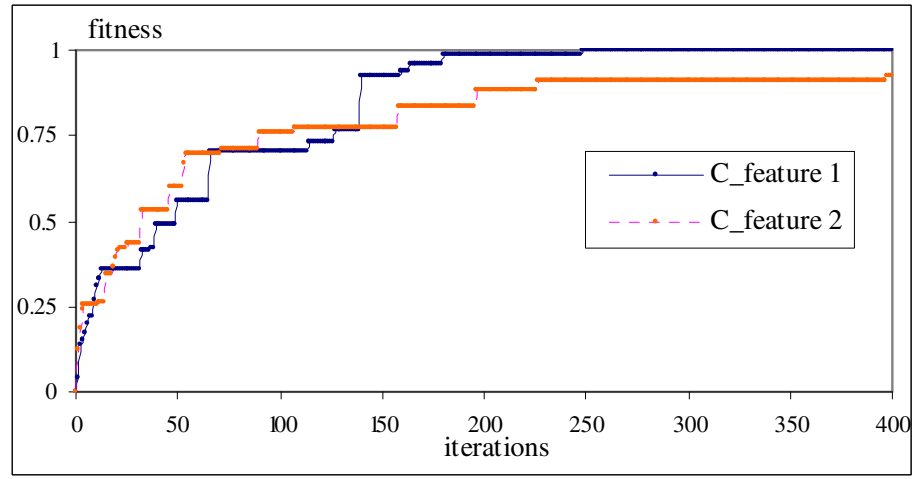

Fig. 5. Convergence results for the features produced by GP-EM-MSE

\subsection{Identification Results}

The goal of the identification is to classify hand images into a known number of 4 categories or classes according to the decision space of the generated features. With the classifier MDC, Table 2 shows the confusion matrix of the identification performance for four classes on the test set, using two features, P_feature 1 and P_feature 2, produced by GP-EM-MSE. Each row of the table represents the identification performance for a given category, while each column represents a percentage of the number of samples in an actual class. It can be observed, from Table 2 , that the difference of the related identification accuracy among the classes is small, ranging from $95.00 \%$ to $96.96 \%$. 
Table 2. Identification accuracy (\%) using two features, $\mathrm{P}_{-}$feature 1 and $\mathrm{P}_{-}$feature 2, produced by GP-EM-MSE with MDC

\begin{tabular}{llcccc}
\hline \multicolumn{2}{c}{ categories } & class I & class II & class III & class IV \\
\hline class I & (33 samples) & 96.96 & 3.04 & 0 & 0 \\
class II & (25 samples) & 0 & 96.00 & 0 & 4.00 \\
class III & (22 samples) & 4.55 & 0 & 95.45 & 0 \\
class IV & (20 samples) & 0 & 5.00 & 0 & 95.00 \\
\hline
\end{tabular}

\subsection{Comparison Results}

The classifiers MDC and KNN are utilized in order to assess the capability of different feature sets over different classification systems. In terms of the identification accuracy, Table 3 shows the comparison results between the features produced by GP-EM-MSE with MDC and 41 primitive features selected by the method [4] with $\mathrm{KNN}$ in which the value of $\mathrm{K}$ is tested by the input of 41 primitive features $(K=41)$.

Table 3. The comparision between the features produced by GP-EM-MSE / MDC and 41 primitive features selected by [4] / KNN, in terms of the identification accuracy (\%)

\begin{tabular}{lcc}
\hline \multicolumn{1}{c}{ feature types / classifier } & no. of features & identification accuracy (\%) \\
\hline the primitive features selected by [4] / KNN & 41 & 93.0 \\
P_feature 1 / MDC & 1 & 92.0 \\
P_feature 1, P_feature 2 / MDC & 2 & 96.0 \\
\hline
\end{tabular}

It can be seen from Table 3 that the identification accuracy using one feature, $\mathrm{P}_{\text {ffeature }} 1$, is slightly lower than when using 41 primitive features. However, the combination of two features, $\mathrm{P} \_$feature 1 and $\mathrm{P} \_$feature 2, produced by GP-EM-MSE achieves the best identification performance with an accuracy rate at $96.0 \%$.

\section{Conclusion and Future Work}

As an extension of the method of CCCA for the feature selection in [4], the purpose of GP-EM-MSE in this study is to find the improved feature representations in an optimal control environment in order to further minimize identification errors for the hand-based biometric system. GP-EM-MSE has succeeded in producing correct features to identify hand images with the improved performance.

The previous work of the CCCA method was evolved without supervision in such a way that the selected feature sets operated globally to cluster hand images. Consequently, the current study of GP-EM-MSE for the feature generation is a supervised learning algorithm, via the results of CCCA. In the case of some not-sosimple problems, the GP-EM-MSE method offers a customized general purpose research platform by jointly optimizing feature representations, which demonstrates that such a combination of methods of the feature selection and generation leads to a higher recognition rate. 
Our future work will be dedicated to examining the robustness of the system in difficult pattern identification problems and large databases of samples.

Acknowledgments. This work is supported by grants from the NSERC.

\section{References}

1. Zheng, G., Wang, C.-J., Boult, T.E.: Application of projective invariants in hand geometry biometrics. IEEE Trans. Information Forensics and Security 2, 758-768 (2007)

2. Zhang, D., Kong, W., You, J.: Online palmprint identification. IEEE Trans. Pattern Anal. Mach. Intell. 25, 1041-1050 (2003)

3. Rizki, M.M., Zmuda, M.A., Tamburino, L.A.: Evolving pattern recognition systems. IEEE Trans. Evol. Comput. 6, 594-609 (2002)

4. Kharma, N., Suen, C.Y., Guo, P.-F.: Palmprints: a cooperative co-evolutionary algorithm for clustering hand images. Int. J. Image and Graphics 5, 595-616 (2005)

5. Guo, P.-F.: Palmprints: a cooperative co-evolutionary clustering algorithm for hand-based biometric identification. M.A.Sc. thesis, Concordia University, Montreal, Canada (2003)

6. Koza, J.R.: Genetic Programming II: Automatic Discovery of Reusable Programs. MIT Pr., Cambridge (1994)

7. Lee, C.-Y.: Efficient automatic engineering design synthesis via evolutionary exploration. Ph.D thesis, California Institute of Technology, Pasadena, California (2002)

8. Raymer, M.L., Doom, T.E., Kuhn, L.A., Punch, W.F.: Knowledge discovery in medical and biological datasets using a hybrid Bayes classifier/evolutionary algorithm. IEEE Trans. Syst., Man, Cybern., Part B 33, 802-813 (2003)

9. Bishop, C.M.: Pattern Recognition and Machine Learning. Springer, New York (2006)

10. Mitchell, T.M.: Machine Learning. McGraw-Hill, New York (1997)

11. Fukunaga, K.: Introduction to Statistical Pattern Recognition, 2nd edn. Academic, Boston (1990) 